\title{
Diagnóstico de la experiencia \\ en línea de docentes y estudiantes \\ de la UAA
}

Norma Isabel Medina Mayagoitia y Juan Jesús Tlapalamatl Esparza

\section{Resumen}

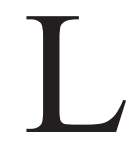

a combinación de los datos estadísticos y las vivencias de la educación a distancia en tiempos de pandemia permiten integrar en este artículo la perspectiva de la comunidad docente y estudiantil de la Universidad Autónoma de Aguascalientes (UAA), ante este suceso inédito en los procesos de enseñanza y aprendizaje. A través de un proyecto de investigación, iniciado previamente al confinamiento, se presentan datos importantes para estructurar un diagnóstico sobre las condiciones institucionales de la educación en línea, con el apoyo de encuestas realizadas a la comunidad universitaria en el primer semestre de esta situación de emergencia sanitaria. Los resultados presentan las ventajas y áreas de oportunidad con respecto a las competencias digitales de docentes, la satisfacción de estudiantes sobre las clases virtuales y los recursos tecnológicos disponibles para continuar los programas educativos de la UAA, a pesar de las circunstancias del momento.

Palabras clave: educación en línea, desempeño docente, satisfacción estudiantil.

\section{Introducción}

Ante la obligatoriedad de la enseñanza en línea por la pandemia de COVID-19, a partir de abril de 2020 ninguna persona de nuestra comunidad universitaria imaginábamos que en unas cuantas semanas se transformarían los procesos educativos presenciales en clases totalmente virtuales durante más de un año.

Este artículo presenta información sistematizada sobre la preparación, aplicación y satisfacción de la educación en línea de la UAA durante los primeros meses de la contingencia, la cual fue recabada por tres departamentos administrativos de la institución. El procesamiento y análisis de los siguientes datos forman parte del diagnóstico de la investigación Condiciones y posibilidades para la UAAVirtual, proyecto desarrollado para ofrecer directri- ces de orientación hacia la creación de un sistema universitario virtual. ${ }^{1}$

En la primera parte se incluyen resultados de la habilitación de una muestra de profesores y profesoras de la UAA en la educación a distancia y el desenvolvimiento que reportaron de abril a junio de 2020 como docentes en línea. El grado de satisfacción de las y los estudiantes respecto a esta modalidad educativa, $\mathrm{y}$ a las competencias digitales de sus docentes, se expone en el segundo apartado, con información recabada seis meses posteriores al inicio de las clases virtuales. Por último, en las conclusiones se hace referencia a la trayectoria institucional de apoyo a la educación en línea y las posibilidades de continuar investigando al respecto.

1 Esta investigación se llevó a cabo por la autora, de enero de 2020 a diciembre de 2021, en la que participó como becario el coautor de este artículo, además de la doctora Rebeca PadiIla, colaboradora del estudio, y la licenciada Fernanda Campos, asistente de investigación. 


\section{Desempeño en línea de los docentes de la UAA}

La emergencia sanitaria y el confinamiento propiciaron el diseño de instrumentos de información específicos que dieran cuenta de la situación que prevalecía entre los grupos de docentes para enfrentarse abruptamente a la educación en línea durante los primeros meses posteriores al periodo vacacional de abril de 2020 .

Unas semanas previas al regreso a clases, el Departamento de Innovación Educativa de la Dirección General de Docencia de Pregrado, con el apoyo de los centros académicos de la UAA, aplicó una encuesta a dis- tancia para identificar el nivel de participación y dominio de docentes en el manejo de Aula Virtual o uso de la plataforma Moodle. Los resultados de esta encuesta permitirían reconocer ventajas y limitaciones para contribuir en su habilitación.

Un total de 418 docentes, responsables de materias de licenciatura, respondieron la encuesta independientemente del tipo de contratación o nombramiento que tenían en el semestre enero-junio de 2020. A partir de la base de datos, proporcionada por el departamento señalado, se elaboró la siguiente gráfica sobre la experiencia que tenían en Aula Virtual.

Gráfica 1. Tipo de participación de docentes de la UAA en Aula Virtual, abril de 2020

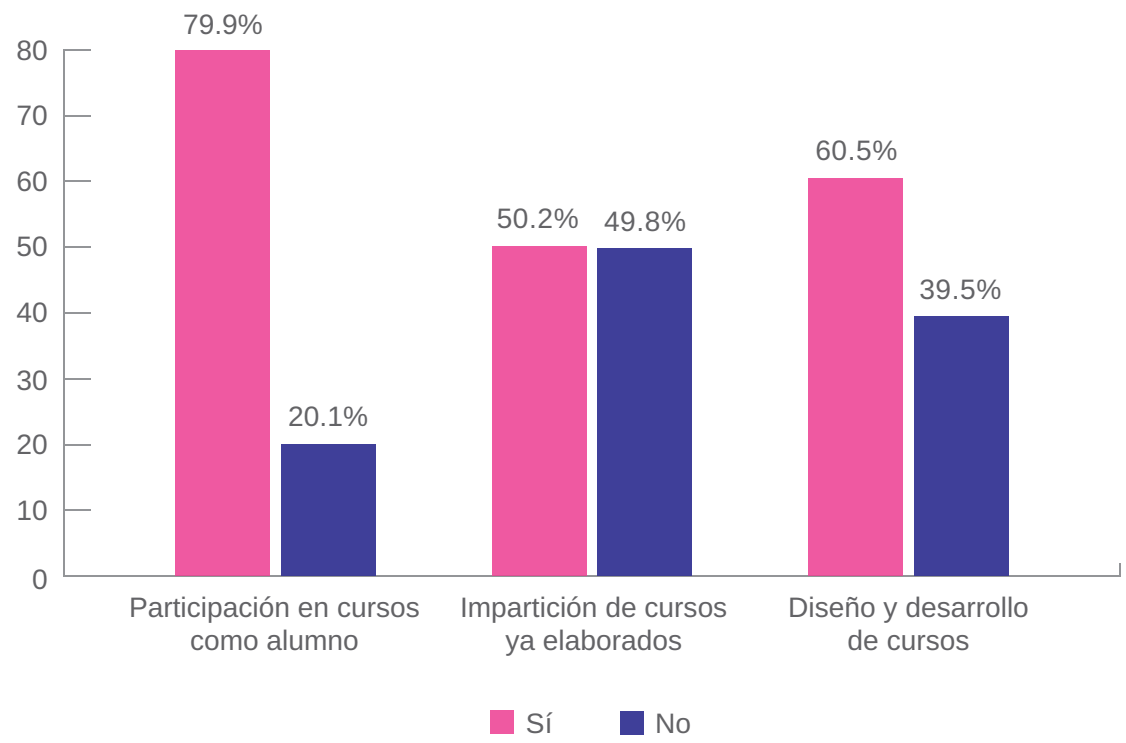

Fuente: Elaborada por los autores a partir de Universidad Autónoma de Aguascalientes, 2020a

Si bien, cerca de $80 \%$ de los encuestados había participado como estudiante en Aula Virtual, lo cual representaba una ventaja para tener empatía al momento de ser docentes a distancia, el hecho de que casi $50 \%$ no hubiera practicado la enseñanza en línea desde su diseño y planeación, y que $39.5 \%$ no tuviera la experiencia de dar clases en esta modalidad impulsó a la preparación e implementación urgente de un programa intensivo de formación. Por otra parte, es alentador que $60.5 \%$ ya se desenvolvían como docentes en línea, aunque requerían actualizarse en el manejo de recursos y herramientas de Aula Virtual, como se muestra en la Gráfica 2, en la que se concentran cuatro aspectos básicos para la enseñanza en la plataforma.
Un porcentaje reducido de docentes, inferior a $8 \%$, manifestó un dominio nulo para colocar instrucciones de actividades de aprendizaje, tareas y foros en Aula Virtual, pero $22.7 \%$ necesitaba aprender a gestionar cuestionarios en línea por ser el recurso que menos sabían utilizar. Estos resultados revelaron un mayor uso de la plataforma como repositorio, y poco aprovechamiento para contenidos en línea de las materias presenciales.

《La emergencia sanitaria y el confinamiento propiciaron el diseño de instrumentos

de información específicos que dieran cuenta de la situación que prevalecía entre los grupos de docentes para enfrentarse abruptamente a la educación en línea》 
Gráfica 2. Nivel de dominio de docentes de la UAA en el manejo de Aula Virtual, abril de 2020

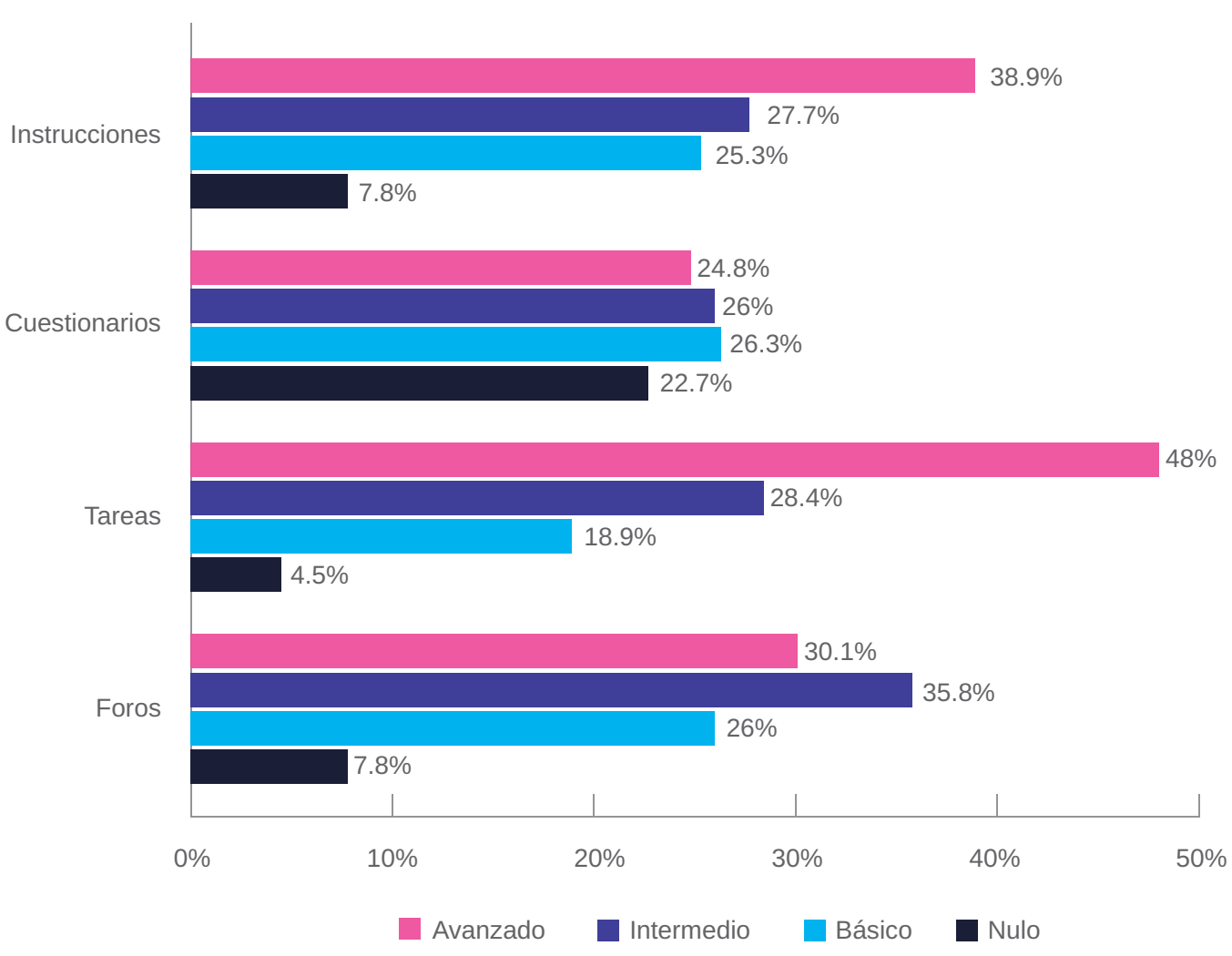

Fuente: Elaborada por los autores a partir de Universidad Autónoma de Aguascalientes, 2020a.

Por otra parte, con el fin de dar cuenta del desarrollo del trabajo a distancia, el Departamento de Evaluación Educativa de la Dirección General de Docencia de Pregrado gestionó la aplicación de un reporte semanal en línea que debía completarse por profesores y profesoras, del 27 de abril al 19 de junio de 2020. Un promedio de 1,512 docentes, de los 10 centros académicos de la UAA, enviaron este reporte semanal en el periodo mencionado.

Respecto a los recursos tecnológicos que se utilizaron en mayor porcentaje para impartir las clases virtuales destacó el Aula Virtual, seguida de WhatsApp, el correo electrónico y Teams. Los cursos de formación de docentes para el manejo de Aula Virtual fortalecieron sus competencias, de ahí que recurrieran principalmente a esta plataforma; sin embargo, una cantidad importante se inclinó por medios más directos para continuar con los programas educativos, incluso el empleo del correo electrónico fue elevado, a pesar de que las y los estudiantes lo han sustituido por las redes sociales. Asimismo, se evidenció un mayor aprovechamiento de Teams hacia el final del semes- tre para tener comunicación sincrónica con los grupos estudiantiles a través de videollamadas, una posibilidad que no ofrece Aula Virtual. En la Gráfica 3 se comparan los resultados correspondientes a la primera semana y la última del reporte en línea mencionado.

Otro aspecto reportado por las y los docentes fueron las dificultades técnicas que surgieron frente a la alta demanda en el uso de tecnologías, sobre todo al inicio, así que $49 \%$ manifestó deficiencias en la conectividad y $16 \%$ señaló fallas en Aula Virtual, cifras que se redujeron al término del semestre. Estos problemas en los servicios domiciliarios de internet de docentes y estudiantes, además de los institucionales, se atendieron para facilitar la continuidad de las clases, aunque no desaparecieron por completo.

Sobre la respuesta estudiantil a la educación en línea, fue calificada entre muy buena y buena ( $43 \%$ y $34 \%$, respectivamente); no obstante, estos resultados decrecieron en la última semana del semestre de dos a seis puntos porcentuales, un indicio del agotamiento del trabajo a distancia. 
Gráfica 3. Principales recursos tecnológicos utilizados para la educación en línea, por docentes de la UAA, abril-junio de 2020

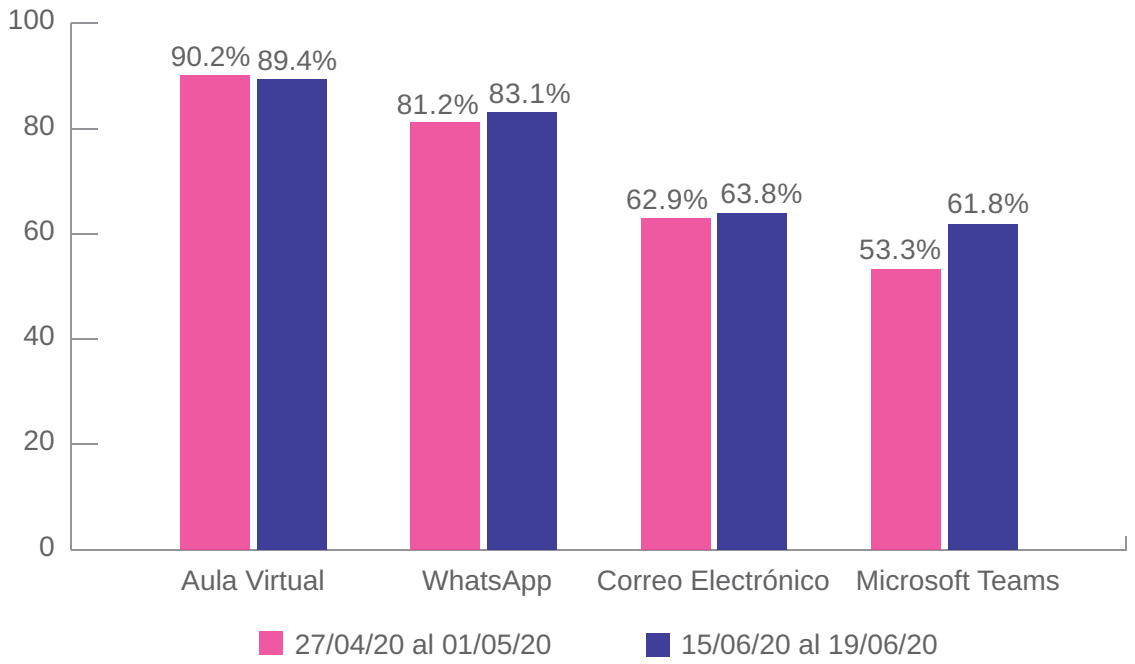

Fuente: Elaborada por los autores a partir de Universidad Autónoma de Aguascalientes, 2020b.

\section{Opiniones sobre la educación en línea de estudiantes de la UAA}

Cada año, la Dirección General de Planeación y Desarrollo, a través del Departamento de Gestión Organizacional y Calidad, aplica al estudiantado la encuesta de satisfacción del usuario. En octubre de 2020 se aplicó esta encuesta en línea a 20,103 estudiantes de bachillerato, licenciatura y posgrado de la UAA, obteniendo una respuesta de $89.4 \%$.

Sobre el proceso de enseñanza y aprendizaje -que por supuesto se había estado llevando a cabo a distancia de abril a la fecha, aunque en la encuesta no se especificara-, $83.85 \%$ de las y los estudiantes manifestaron sentirse muy satisfechos o satisfechos, mientras que $16.15 \%$ poco satisfechos o totalmente insatisfechos. De igual manera, en cuanto a las habilidades digitales de docentes, el grado de satisfacción registrado fue alto, como se puede apreciar en la Gráfica 4.

《Sobre la respuesta estudiantil a la educación en línea, fue calificada entre muy buena y buena ( $43 \%$ y $34 \%$, respectivamente); no obstante, estos resultados decrecieron en la última semana del semestre de dos a seis puntos porcentuales, un indicio del agotamiento del trabajo a distancia $\gg$
Gráfica 4. Satisfacción de estudiantes de la UAA sobre habilidades de docentes en el uso y aplicación de recursos virtuales, octubre de 2020

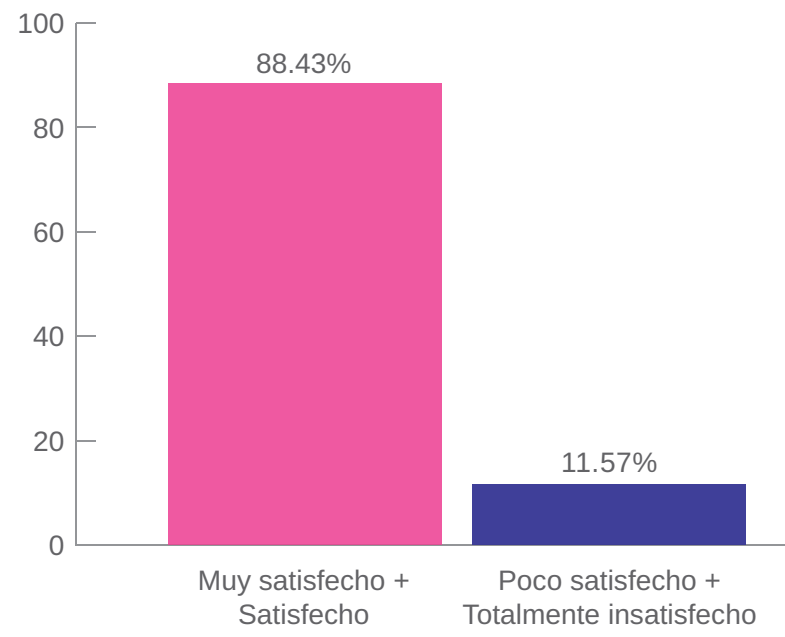

Fuente: Elaborada por los autores a partir de Universidad Autónoma de Aguascalientes, 2020c.

Los porcentajes anteriores no coinciden totalmente con las experiencias narradas durante las sesiones en línea; de hecho, al final de la encuesta hubo quienes agregaron comentarios sobre las deficiencias de la educación en línea, el disgusto por las clases virtuales, la falta de competencias docentes, así como las fallas técnicas y de conectividad en Aula Virtual y en sus hogares. Frases como "Espero con ansias desinstalar Teams de mi computadora" y otras más, son ejemplos de algunas de las posturas estudiantiles en torno a la educación a distancia. 


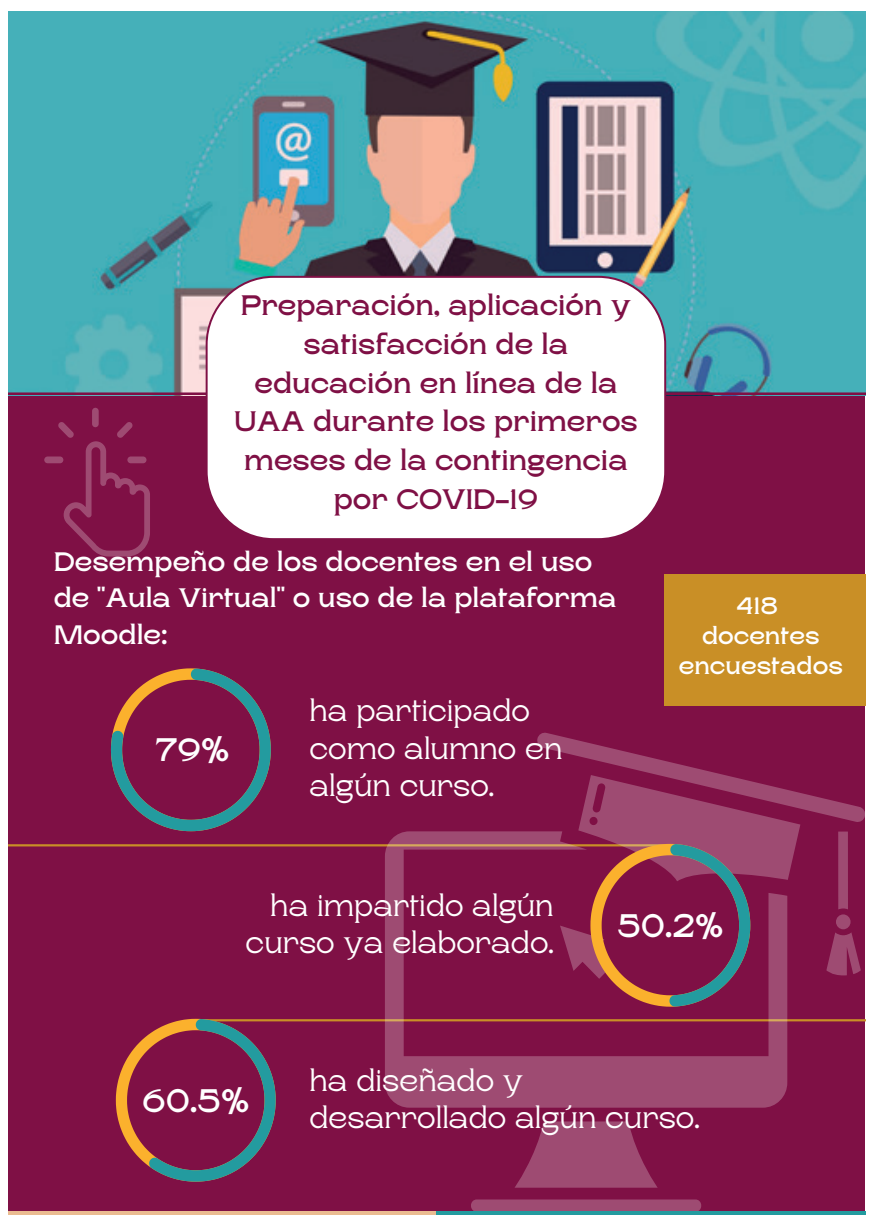

Principales recursos tecnológicos utilizados para la educación en línea:

1
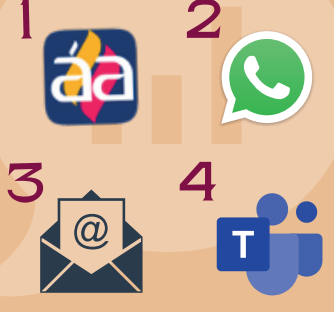

Dificultades técnicas:

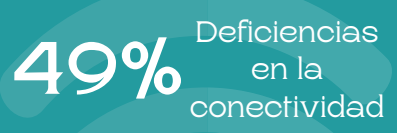

Fallas en el "Aula Virtual"

\section{Conclusiones}

Los resultados nos muestran un panorama preliminar sobre el desarrollo de la modalidad educativa en línea de la UAA en el contexto de la pandemia, con sus ventajas y limitaciones. Sin embargo, es importante mencionar que la institución ha realizado desde décadas atrás las siguientes acciones para fortalecer la educación a distancia: 1) formación y actualización docente a través de cursos y diplomados, con una trayectoria de más de 15 años a la fecha; 2) disposición de espacios en Aula Virtual de todas las materias curriculares a partir de 2012; 3) oferta de cursos y diplomados en línea para la educación continua abierta a profesionistas; 4) inversión permanente en proyectos de infraestructura tecnológica; y 5) apoyo especializado de soporte técnico en beneficio de las clases virtuales. Todo esto contribuyó no sólo para afrontar la crisis sanitaria, sino para avanzar en la aplicación de metodologías de enseñanza y aprendizaje alternativas con el uso de las TIC, lo cual nos permite reflexionar sobre las posibilidades de aprovecharlas para atender circunstancias particulares, además de promover el acceso a la educación de nivel superior.

Si bien en este artículo se destaca la situación respecto a la docencia y se evidencia la necesidad de recuperar mayor información de los grupos estudiantiles, en próximas fechas se contará con más datos y a mayor profundidad sobre los aspectos a favor y en contra de la educación en línea, así como las probabilidades de disponer de un campus virtual a futuro, de acuerdo a la perspectiva de la comunidad universitaria, mediante las investigaciones en proceso que se llevan a cabo en la UAA.

\section{Fuentes de consulta}

Universidad Autónoma de Aguascalientes [UAA]. (2020a).

Satisfacción de la educación en línea por parte de los estudiantes:

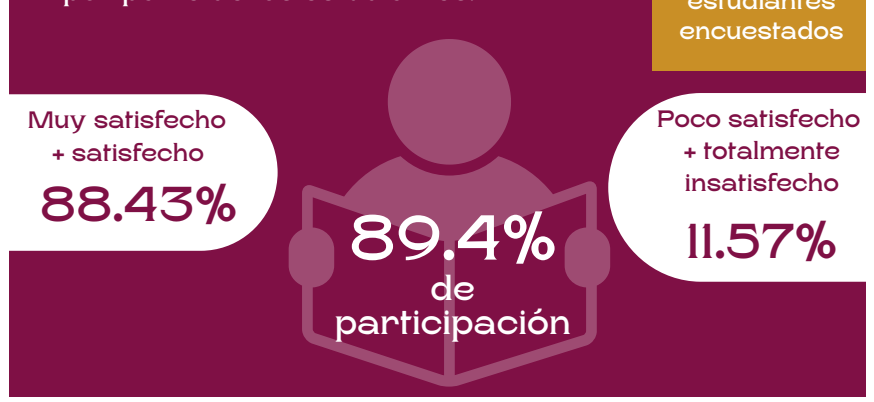

20,103 estudiantes encuestados oco satisfecho + totalmente $1.57 \%$ Base de datos y resultados de encuesta aplicada a docentes en abril de 2020. [Documento de trabajo]. UAA-DGDP-DIE.

UAA. (2020b). Resultados de reportes semanales de docentes de los centros académicos de la UAA. [Documento de trabajo]. UAA-DGDP-DEE.

UAA. (2020c). Resultados de la Encuesta de satisfacción del usuario 2020. [Documento de trabajo]. UAADGPD-DGOC.

Infografía elaborada por el Departamento de Formación y Actualización Académica (2021) 